\title{
The noncanonical BMP signaling pathway plays an important role in club cell regeneration
}

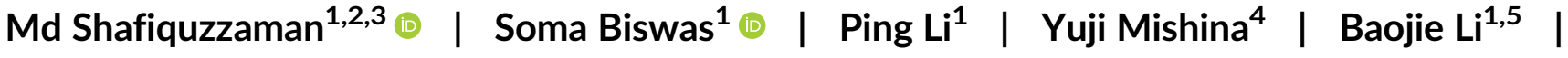 \\ Huijuan Liu ${ }^{1,2}$
}

${ }^{1}$ Bio-X Institutes, Key Laboratory for the Genetics of Developmental and Neuropsychiatric Disorders, Ministry of Education, Shanghai Jiao Tong University, Shanghai, People's Republic of China

${ }^{2}$ Metabolic Bone Disease and Genetics Research Unit, Department of Osteoporosis and Bone Diseases, Shanghai Jiao Tong University Affiliated Sixth People's Hospital, Shanghai, People's Republic of China

${ }^{3}$ National Institute of Biotechnology, Ministry of Science and Technology, Dhaka, Bangladesh ${ }^{4}$ Department of Biologic \& Materials Sciences, School of Dentistry, University of Michigan, Ann Arbor, Michigan

${ }^{5}$ Center for Traditional Chinese Medicine and Stem Cell Research, The Chengdu University of Traditional Chinese Medicine, Sichuan, People's Republic of China

\section{Correspondence}

Huijuan Liu, PhD, Bio-X Institutes, Shanghai Jiao Tong University, Shanghai 200240,

People's Republic of China.

Email: liuhj@sjtu.edu.cn

Funding information

Grants from the State Key Laboratory of Oncogenes and Related Genes, Grant/Award Number: 90-17-03; National Key Scientific Program, Grant/Award Numbers: 2017YFA0103602, 2018YFA0800803; National Natural Science Foundation of China, Grant/Award Numbers: 81130039, 81421061

\begin{abstract}
The bronchiole is a major site for the development of several life-threatening disorders, including chronic obstructive pulmonary disease and lung adenocarcinomas. The bronchiolar epithelium is composed of club cells and ciliated epithelial cells, with club cells serving as progenitor cells. Presently, the identity of the cells involved in regeneration of bronchiolar epithelium and the underlying mechanisms remain incompletely understood. Here, we show that Prrx1, a homeobox transcription factor, can mark club cells in adult mice during homeostasis and regeneration. We further show that the noncanonical signaling pathway of BMPs, BMPR1ATak1-p38MAPK, plays a critical role in club cell regeneration. Ablation of Bmpr1a, Tak1, or Mapk14 (encoding p38 $\alpha$ ) in Prrx $1^{+}$club cells caused minimal effect on bronchiolar epithelium homeostasis, yet it resulted in severe defects in club cell regeneration and bronchiole repair in adult mice. We further show that this pathway supports proliferation and expansion of the regenerating club cells. Our findings thus identify a marker for club cells and reveal a critical role for the BMP noncanonical pathway in club cell regeneration.
\end{abstract}

\section{KEYWORDS}

bronchiolar epithelium, club cell regeneration, club cells, noncanonical BMP pathway, Prrx1, Tak1-p38MAPK

\section{1 | INTRODUCTION}

The respiratory system is developed from the ventral foregut endoderm cells marked by Nkx2-1 in mouse. ${ }^{1,2}$ By E16.5, the lung buds form a tree-like architecture of airways with thousands of terminal tubules, ${ }^{3}$ which generate numerous epithelial sacs that later develop into alveoli. ${ }^{4}$ In adult mice, the respiratory system displays low turnover rate. ${ }^{2}$ Yet, it has great regeneration potentials in response to injuries caused by influenza virus infection, toxic chemical inhalation or other insults. In trachea, $\mathrm{p} 3^{+}$basal cells can regenerate ciliated and secretory epithelial cells. ${ }^{5}$ In small airways (bronchioles), Scgb1a1 ${ }^{+}$(Uteroglobin) club cells self-renew and give rise to ciliated cells and contribute to repair of bronchiolar epithelium. ${ }^{6-9}$ Earlier studies have suggested that club cell population is heterogenous and a portion of Scgb1a1 $1^{+}$club cells, termed variant club cells, is stem cells. ${ }^{8,10,11}$ Most recently, Upk3a has also been proposed to identify a rare subset of club cells with differentiation potentials. ${ }^{12}$ In alveoli, type II cells (AEC2) act as stem cells to produce both type I and II alveolar cells. ${ }^{13,14}$

The BMP family members play key roles in lung development and homeostasis. ${ }^{4,15-19}$ The signal transduction cascade is initiated when BMPs bind to BMP receptor I (BMPRI) and II (BMPRII), which are serine/threonine kinases. ${ }^{20}$ The constitutively active BMPRII then 
phosphorylates and activates BMPRI, which in turn phosphorylates Smad 1/5/8. Smad 1/5/8 associates with Smad4 and translocates to the nucleus where they function as transcription activator or repressor. Previous studies have shown that BMP4 regulates branching morphogenesis and proximal-distal differentiation of lung endoderm. ${ }^{15,19}$ Ectopic expression of BMP4 or deletion of BMP antagonists in lung epithelium causes defects in lung development, ${ }^{17,19}$ whereas inactivation of Bmpr1a and Bmpr1b results in tracheal agenesis. ${ }^{16}$ In addition, Smad1 knockout causes defects in lung branching and epithelium proliferation and differentiation. ${ }^{21,22}$

BMPs also activate noncanonical pathways that include Tak1 and MAPKs. ${ }^{23-25}$ During mouse lung development, Mapk14 (encoding p38 $\alpha$ ) is expressed as early as E10.5 and is involved in budding/ branching, proliferation, and differentiation of epithelial progenitor cells at the distal tips of the endoderm. ${ }^{26}$ Ablation of Mapk14 caused neonatal lethality, accompanied by abnormal alveolar structures and massive deposition of hematopoietic cells in the lung. ${ }^{27}$ Postnatal deletion of Mapk14 in the whole animal impaired proliferation and differentiation of lung stem and progenitor cells. ${ }^{28}$

In addition to controlling embryonic lung development, BMP signaling pathway also regulates postnatal lung homeostasis and repair. $^{29,30}$ A recent study showed that inhibition of BMP signaling promoted basal cell proliferation but not cell fate determination whereas BMP4 inhibited both basal cell proliferation and differentiation. $^{31}$ BMPs also play similar roles in AEC2 in response to pneumonectomy, ${ }^{32}$ where BMPs keep stem cells in a quiescent state. A transient upregulation of BMP inhibitors during repair promotes cell proliferation and facilitates the repair process. However, the functions of the BMP noncanonical pathways in lung homeostasis and regeneration are less investigated and remain elusive.

Club cells secrete uteroglobin, surfactant, and other proteins to protect the bronchiolar lining cells and to detoxify inhaled harmful substances, whereas the ciliated cells pump fluid, which carries dust and germs, in or out of the bronchiolar lumen. Moreover, the bronchial and bronchioles are the major sites for the development of chronic obstructive pulmonary disease and lung adenocarcinomas. ${ }^{33,34}$ Yet, the identity of the progenitor club cells and the mechanisms by which these cells regenerate bronchiolar epithelium remain incompletely understood. Here, our lineage tracing studies and conditional gene knockout mouse experiments identify Prrx1 as a marker for club cells and provide genetic evidence that the BMPR1A-Tak1-p38MAPK pathway plays an indispensable role in regeneration of bronchiolar epithelium by promoting club cell proliferation and expansion.

\section{2 | MATERIALS AND METHODS}

\section{$2.1 \mid$ Mice}

The Rosa-tdTomato and the transgenic Prrx1-Cre mice were purchased from the Jackson Laboratory. They were mated to obtain the Prrx1-Cre; Rosa-tdTomato mice. The transgenic Prrx1-CreERT mice were generated in Shunichi Murakami's lab, ${ }^{35}$ the floxed Bmpr1a mice were generated in Yuji Mishina's lab, ${ }^{36}$ and $T a k 1^{f / f}$ mice were generated in Michael

\section{Significance Statement}

Club cells are the stem cells in the airways that are capable of self-renewal and differentiation to other cells. However, markers for these cell populations and the signaling pathways involved in their stem cell activities are not well understood. To the best of authors' knowledge, this study reports for the first time that the BMPR1A-mediated Tak1p38MAPK pathway is indispensable for club cell regeneration and repair of bronchiolar epithelium. Furthermore, Prrx1 was also identified as a marker for club cells in the adult mouse lung.

D. Schneider's lab. Bmpr1 $1 a^{f / f}$ and Tak $1^{f / f}$ mice were crossed with Prrx1-CreERT mice to generate Prrx1-CreERT; Bmpr1 $a^{f / f}$ and Prrx1-CreERT; Tak1 ${ }^{f / f}$ mice, in which Bmpr1a and Tak1 were deleted after tamoxifen administration, respectively. The Mapk14 ${ }^{f / f}$ mouse line was generated in Dr. Yibin Wang's laboratory at UCLA and it was bred with the Prrx1-Cre mice to get the Prrx1-Cre; Mapk14 f/f mice. The C57BL6 mice were purchased from the Charles River Laboratories.

All animals were bred and housed in the specific pathogen-free (SPF) facility of Bio-X institutes, Shanghai Jiao Tong University, and food and water were provided ad libitum. Animal experimentations in this study were performed following the recommendations of the National Research Council Guide for Care and Use of Laboratory Animals, with all the experimental protocols approved by the Institutional Animal Care and Use Committee of Shanghai Jiao Tong University, China (SYXK [SH] 2011-0112).

\section{2 | Naphthalene (NA) administration}

NA (Sigma-Aldrich, St. Louis, Missouri, https://www.sigmaaldrich.com/ catalog/product/sial/84679?lang $=\mathrm{en}^{\circledR}$ ion=US) was dissolved in corn oil and a single dose was administered by i.p. injection $(280 \mathrm{mg} / \mathrm{kg}$ body weight). Lung tissues were collected on day 3 and 7 after NA treatment and processed for analyses.

\section{3 | Tamoxifen (TAM) administration}

TAM (T5648, Sigma-Aldrich, St. Louis, Missouri) powder was dissolved in corn oil at a concentration of $10 \mathrm{mg} / \mathrm{mL}$ and was kept foil-wrapped to protect from light. TAM was administered i.p. at a dose of $100 \mathrm{mg} /$ $\mathrm{kg}$ body weight on every other day for a total of three doses.

\section{4 | Tissue processing and histology}

Mice were anesthetized by administration of avertin $(2.5 \%$ tribromoethanol) through i.p. injection. Chest cavity was opened surgically and $0.9 \%$ normal saline was used for perfusion through both left and right ventricle. The trachea was ligated after inflating the lungs with $1.5 \mathrm{~mL}$ of $4 \%$ paraformaldehyde (PFA, $\mathrm{pH} 7.4$ ) in PBS through the trachea. The heart and lungs were removed en bloc and 
further fixated overnight in $4 \%$ PFA at $4^{\circ} \mathrm{C}$. For cryosections, superior lobe of the right lung was removed after overnight fixation and embedded in OCT after dehydrating in graded sucrose solution and cryomicrotome (Leica CM3050S) was used to cut $4 \mu \mathrm{m}$ thick sections. For paraffin sections, the left lung was removed, dehydrated in graded ethanol series, cleared in xylene, embedded in paraffin, and $4 \mu \mathrm{m}$ thick sections were cut using Leica RM2255 microtome.

\section{5 | Immunofluorescence}

Paraffin sections were deparaffinized in xylene and rehydrated in graded ethanol series. Antigen retrieval was performed using $10 \mathrm{mM}$ sodium citrate buffer $(\mathrm{pH} 6.0)$. Tissue sections were permeabilized with $0.1 \%$ Triton $\mathrm{X}-100$ for 30 minutes at room temperature (RT), blocked with $10 \%$ goat serum (in PBS) for 1 hour at RT, and then incubated overnight with primary antibodies at $4^{\circ} \mathrm{C}$. On the next day, tissue sections were incubated with secondary antibodies at $37^{\circ} \mathrm{C}$ for 1 hour and then mounted using SlowFade Gold antifade mountant (Invitrogen). DAPI (Invitrogen) was used to stain the nuclei. Primary antibodies used in this study are: Scgb1a1 (Abcam, ab40873, 1:750), $\alpha$-acetylated Tubulin (Sigma-Aldrich, T7451, 1:600), and Ki67 (Abcam, ab15580, 1:100). Secondary antibodies used are: Alexa Fluor 488-goat anti-rabbit and Alexa Fluor 546-goat anti-mouse (both from Invitrogen, 1:100). Images were captured using Nikon Eclipse 80i microscope (Nikon Instruments Inc.).

\section{6 | Immunohistochemistry}

Paraffin sections prepared as described in the previous section were treated with $3 \% \mathrm{H}_{2} \mathrm{O}_{2}$ in Methanol for 30 minutes in the dark at RT to inactivate endogenous peroxidase. After antigen retrieval, permeabilization, and blocking with $10 \%$ goat serum (in PBS), the sections were incubated overnight with primary antibodies at $4{ }^{\circ} \mathrm{C}$ and then incubated for 1 hour at RT with the secondary anti-Rabbit HRP (Boster). The slides were developed using DAB chromogen (Boster) according to the manufacturer's instructions. Nuclei were counterstained with hematoxylene. Primary antibodies used are: p-Smad 1/5/8 (CST-9511), p-Tak1 (CST-4531), p-p38 (CST-9211) (all from Cell Signaling Technology, 1:50), Scgb1a1 (Abcam, ab40873, 1:750), and $\alpha$-acetylated Tubulin (Sigma-Aldrich, T7451, 1:600). Images were captured using Olympus DP72 microscope (Olympus Microsystems).

\section{7 | Quantification of bronchiolar epithelial cells}

The lung sections prepared for immunofluorescence microscopy were used to quantify bronchiolar epithelial cells. For each section from one mouse, eight fields with different terminal bronchioles were selected randomly and imaged. To quantify Scgb1a1 ${ }^{+}$club cells, Scgb1a1 $1^{+}$cells and all nuclei (counterstained with DAPI) on bronchiolar epithelium in each field were enumerated using ImageJ, scored as ratio of Scgb1a1 $1^{+}$club cells/total bronchiolar epithelial cells, and then averaged to get the final ratio for that mouse. $\alpha$-Tubulin ${ }^{+}$ciliated cells and $\mathrm{Ki} 67^{+}$proliferating cells were counted in the same way. This procedure was followed for all the genotypes used in this study.

\subsection{Quantitative real-time PCR (qPCR)}

Total RNA from lung tissue was extracted with Trizol reagent (Invitrogen) following the manufacturer's instructions. Transcriptor First Strand cDNA synthesis kit (Roche, Basel, Switzerland) with random anchored-oligo (dT) 18 primers and random hexamer was used to synthesize cDNA. qPCR analyses were performed using FS Universal SYBR Green Master Premix (Roche). Reactions were run in triplicate (repeated three times independently) in a total volume of $20 \mu \mathrm{L}$ with Light Cycler 480 Instrument II (Roche). Delta-delta Ct ( $\Delta \Delta C t$ ) method was employed to quantify the relative expression levels of mRNA species. GAPDH (glyceraldehyde 3-phosphate dehydrogenase) served as endogenous control gene to normalize the expression of all target genes. The primer sequences used for real-time PCR are listed below:

$\begin{array}{ll}\text { Bmpr1a } & \text { F: 5'-TCATGTTCAAGGGCAGAATCTAGA-3' } \\ & \text { R: 5'-GGCAAGGTATCCTCTGGTGCTA-3' } \\ \text { Tak1 } & \text { F: 5'-CTGCCAGTGAGATGATCG-3' } \\ & \text { R: 5'-CAGGCTCCATACAACTTGAC-3' } \\ \text { Gapdh } & \text { F: 5'-CCACAGTCCATGCCATCAC-3' } \\ & \text { R: 5'-CATACCAGGAAATGAGCTTGAC-3' }\end{array}$

\section{9 | Western blot analysis}

The lung tissue was homogenized in T-PER reagent (Thermo Fisher Scientific) containing added protease and phosphatase inhibitors. Protein concentration was determined using a BCA Protein Assay Kit (Thermo Fisher Scientific). A $20 \mu$ g of protein lysates of each sample was subjected to SDS-PAGE (8\%-12\%) and transferred onto a nitrocellulose membrane. Immunoblots were then incubated with primary antibodies at $4^{\circ} \mathrm{C}$ with gentle shaking overnight. After being washed with TBST (Tris-buffered saline, 0.1\% Tween 20) three times for 15 minutes each, the blots were incubated with horseradish peroxidase (HRP)-conjugated secondary antibody (Sigma) for 1 hour. Next, the membrane was developed with Super Signal West Femto Maximum Sensitivity Substrate (Thermo Fisher Scientific) and visualized by FluorChem E system (Protein Simple, California). The following antibodies were used: $\mathrm{p}$-Smad 1/5/8 (CST-9511), Smad 1 (CST-9743), p-Tak1 (CST-4531), Tak1 (CST-4505), p-p38 (CST-9211), p38 (CST-9212) (all from Cell Signaling Technology, 1:1000), and $\beta$-Actin (Santa Cruz, Sc-47778, 1:1000).

\subsection{0 | Statistical analyses}

Results were expressed as means \pm SD. Comparisons between wildtype and transgenic mice were analyzed using two-tailed unpaired Student's $t$ test and a $p$ value $<.05$ was considered to indicate statistical significance $\left({ }^{*} p<.05,{ }^{* *} p<.01\right.$, and ${ }^{* * *} p<.001$ when mutant mice were compared with control mice). Each experiment was repeated 3-4 times independently using materials from different mice to confirm the reproducibility of the experimental findings. 


\section{RESULTS}

\section{1 | Prrx1-marked club cells during development and regeneration}

Prrx1 is a marker for mesodermal lateral plate and a transgenic Prrx1-Cre mouse line has been generated to label both osteoblast and chondrocytes during bone development. ${ }^{37-39}$ Intriguingly, we found that in Prrx1-Cre; Rosa-tdTomato mice, tdTomato (tdTO) also labels lung cells. Immunostaining for cell-specific markers on the lung sections of 2-month-old Prrx1-Cre; Rosa-tdTomato mice revealed that tdTomato labeled most club cells $\left(\mathrm{Scgb} 1 \mathrm{a} 1^{+}\right)$, most ciliated cells ( $\alpha$-acetylated Tubulin ${ }^{+}$, hereinafter referred to as $\alpha$-Tubulin), and very few fibroblasts (PDGFR $\alpha^{+}$) (Figure 1A, data not shown). Earlier studies reported that Prrx $1^{-/-}$newborns died from respiratory distress. ${ }^{40,41}$
A

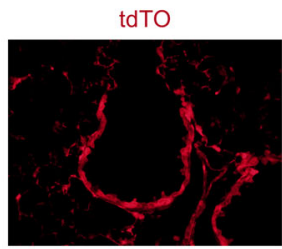

tdTO
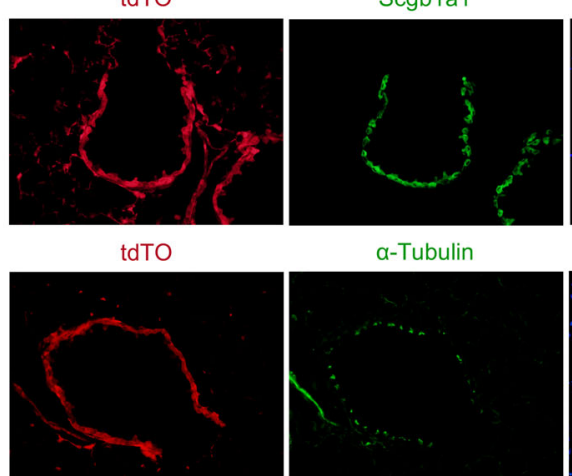

a-Tubulin

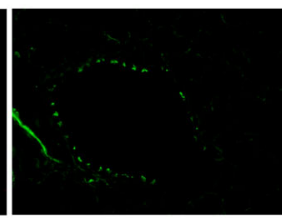

B

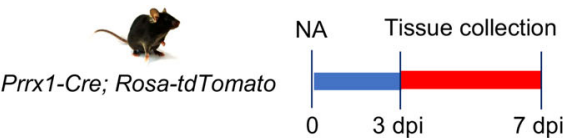

C

tdTO

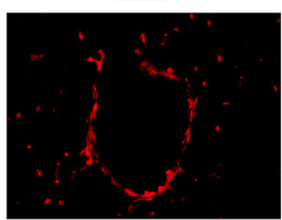

D

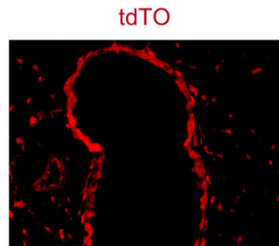

$\mathbf{E}$

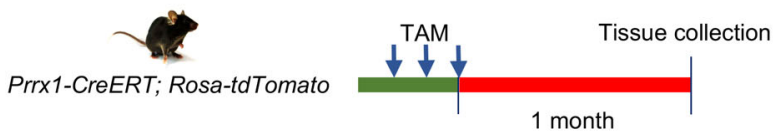

$\mathbf{F}$
$\operatorname{tdTO}$

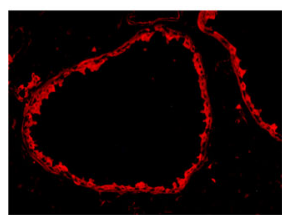

tdTO

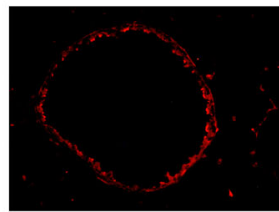

Scgb1a1

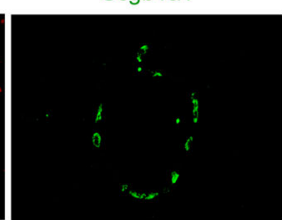

Scgb1a1

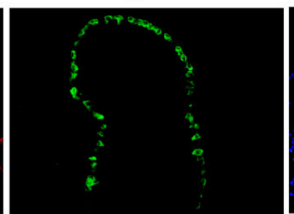

DAPI

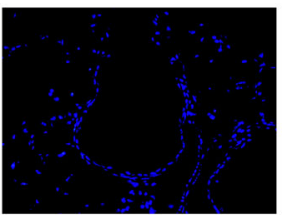

DAPI

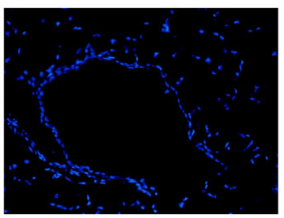

Merge

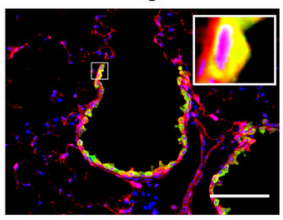

Merge

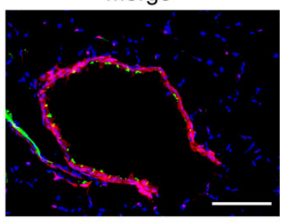

DAPI

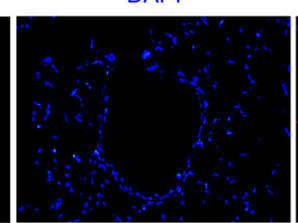

DAPI

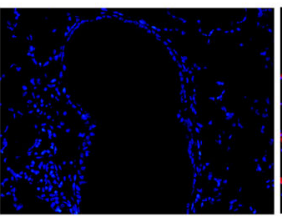

DAPI

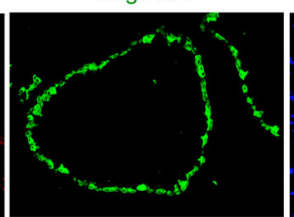

a-Tubulin

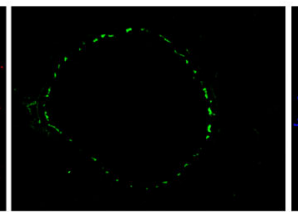

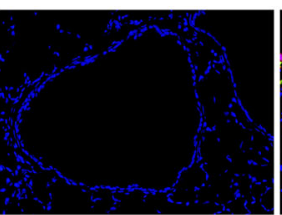

DAPI

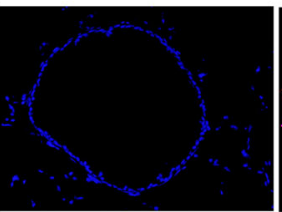

Merge

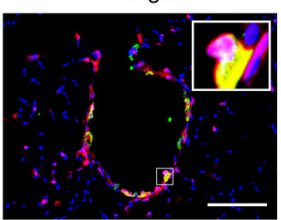

Merge

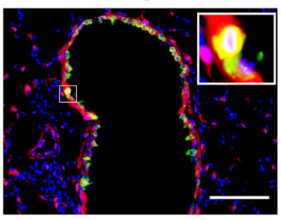

FIGURE 1 Prrx1-labeled club cells in the lung. A, Two-month-old Prrx1-Cre; Rosa-tdTomato mice were used for in vivo tracing of Prrx1 lineage. Immunostaining was performed on lung sections with Scgb1a1 (top panel) and $\alpha$-Tubulin (bottom panel) for club cells and ciliated cells, respectively. Nuclei were counterstained with DAPI (blue). Inset, enlarged view of indicated region showing a representative tdTomato ${ }^{+}$ Scgb1a1 ${ }^{+}$club cell. Scale bar $=50 \mu \mathrm{m}$. $B$, Schedule of naphthalene (NA) administration and tissue collection to Prrx1-Cre; Rosa-tdTomato mice. NA was administered through i.p. injection and the lungs were harvested after 3 or 7 days and then stained with club cell marker Scgb1a1. C,D, Regenerating club cells retained the tdTomato labeling (C) 3 day and (D) 7 day after NA treatment, confirming their Prrx1 lineage. Inset, enlarged view of indicated region. Scale bar $=50 \mu \mathrm{m}$. E, Schematic of TAM treatment to 2-month-old Prrx1-CreERT; Rosa-tdTomato mice. F, Immunostaining of Scgb1a1 (top panel) and $\alpha$-Tubulin (bottom panel) on lung sections of Prrx1-CreERT; Rosa-tdTomato mice. Nuclei were counterstained with DAPI. Scale bar $=50 \mu \mathrm{m}$. dpi, day post injury; tdTO, tdTomato 
Since Prrx1 mainly marks club cells in the bronchioles, there is a likelihood that respiratory distress observed in Prrx $1^{-/-}$newborns may have contributions from the bronchioles.

To examine the behavior of Prrx1 lineage cells during bronchiole regeneration and repair, we adopted a NA-induced lung injury model. NA is metabolized by CYP-2F2, which is exclusively expressed in club cells, generating cytotoxic epoxide which kills most of the club cells within 1.5 days. Hyperplastic growth occurs at day 3 and the bronchiolar epithelium recovers at day $7 .^{42}$ We exposed the Prrx1-Cre; Rosa$t d$ Tomato mice to one dose of NA and the mice were euthanized 3 or 7 days later (Figure 1B). Immunostaining indicated that regenerating club cells $\left(\mathrm{Scgb}_{\mathrm{a}} \mathrm{1}^{+}\right)$were tdTomato positive at day 3 or 7 (Figure $1 \mathrm{C}$,
D), suggesting that the Prrx $1^{+}$lineage cells also participate in bronchiole regeneration/repair.

\section{2 | Prrx1 could mark club cells in adult mice}

We further traced the Prrx1 lineage cells in adult mice using a tamoxifen (TAM)-inducible Prrx1-CreERT mouse line. ${ }^{35}$ TAM was injected into 2-month-old Prrx1-CreERT; Rosa-tdTomato mice with a total of three doses. The mice were euthanized 1 month after the last TAM injection (Figure 1E). Immunostaining results showed that Prrx1 labeled most of the Scgb1a1 $1^{+}$club cells and $\alpha$-Tubulin ${ }^{+}$ciliated cells (Figure 1F), suggesting that Prrx1-CreERT mouse can be used to

A

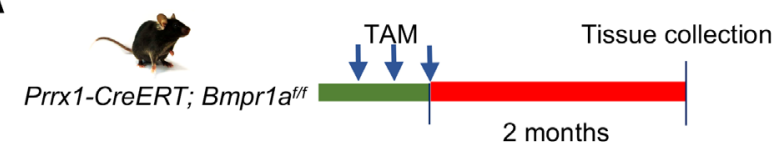

B

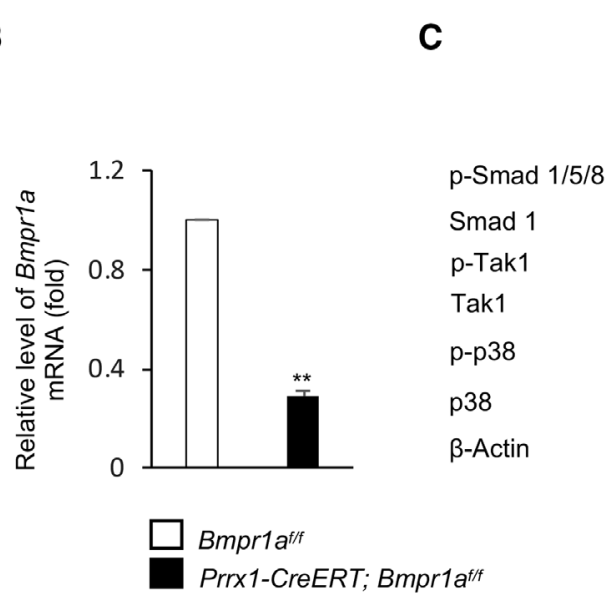

$\mathbf{E}$

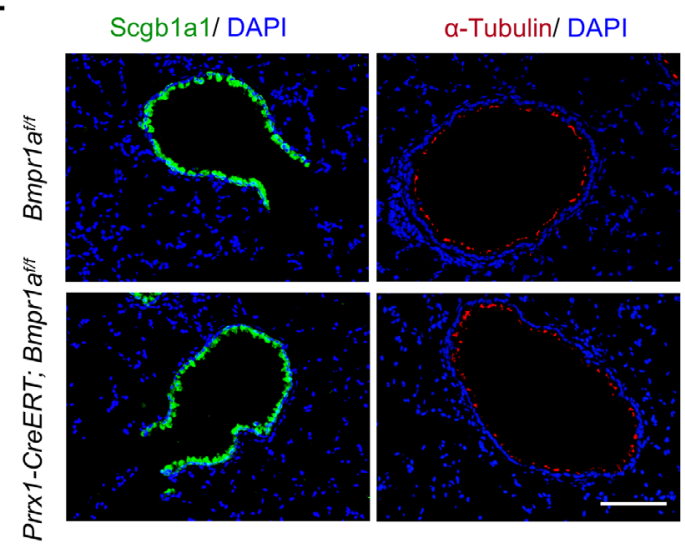

D

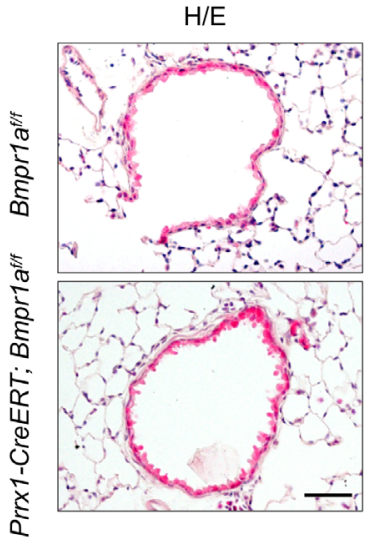

$\mathbf{F}$

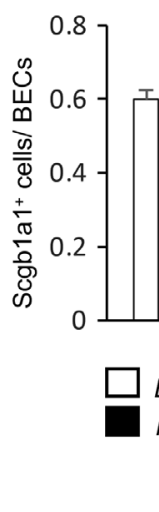

G

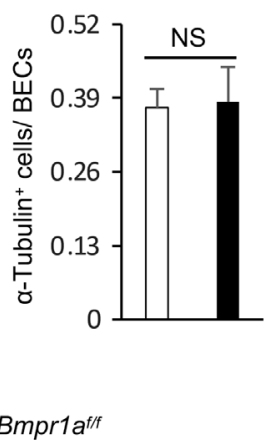

FIGURE 2 Depletion of Bmpr1a produced minimal effects on homeostatic bronchiolar epithelium. A, Schematic of TAM treatment to 2-month-old Prrx1-CreERT; Bmpr1 $a^{f / f}$ and Bmpr1a $a^{f / f}$ (control) mice. B, Successful deletion of Bmpr1a gene after TAM injection was confirmed by qPCR analysis ( $n=6$ mice for each genotype). ${ }^{* *} p<.01$. C, Western blot detected considerable decreases in the levels of $p-S m a d 1 / 5 / 8, p-T a k 1$, and p-p38 in the Bmpr1a deleted lungs compared with the control. D, Histological examination of Bmpr1a knockout and control lung sections revealed little difference. Scale bar $=50 \mu \mathrm{m}$. E, Immunostaining of Scgb1a1 (left panel) and $\alpha$-Tubulin (right panel) on lung sections of mutant and control mice 2 months after TAM administration. Nuclei were counterstained with DAPI. Scale bar $=50 \mu \mathrm{m}$. F,G, Quantification of Scgb1a1 ${ }^{+}$cells (F) and $\alpha$-Tubulin ${ }^{+}$cells (G) per total bronchiolar epithelial cells (BECs). For each lung section, eight different fields were quantified and averaged ( $\mathrm{n}=6$ mice for each genotype). ${ }^{*} p<.05$. NS, not significant 
transiently label club and ciliated cells in adult mice. Overall, these findings prove that Prrx 1 is expressed by the club cells in adult lungs.

\section{3 | The BMP-Tak1-p38MAPK pathway was activated in bronchiolar cells}

BMP signaling pathways are well-established regulators of lung development and lung regeneration/repair mediated by basal cells and AEC2. ${ }^{31,32}$ A previous study has reported that BMP-Smad1 signaling is activated in club cells in response to NA-induced lung injury. ${ }^{43} \mathrm{We}$ found that the Tak1-MAPKs pathway was active in bronchiolar epithelial cells under homeostatic conditions as demonstrated by immunohistochemistry (Figure S1A). To determine whether this pathway is also activated during repair/regeneration, we exposed C57BL6 mice to NA and harvested the lungs after 3 or 7 days. Immunohistochemistry revealed that Tak1 and p38MAPKs were also activated in the bronchiolar epithelium at both time points after NA exposure
(Figure S1A). The levels of p-Tak1 and p-p38 showed considerable increase at day 3 after NA injury. At day 7, when the repair of bronchiolar epithelium is thought to reach completion, their levels decreased. Overall, these results suggest that the noncanonical BMP pathway is activated in bronchiolar epithelial cells under homeostatic conditions and during regeneration.

\section{4 | Bmpr1a deletion in Prrx1 ${ }^{+}$cells caused minor defects in bronchiolar homeostasis}

To evaluate the functions of BMP signaling pathway in maintenance of bronchiolar epithelium, we generated Prrx1-CreERT; Bmpr1 $a^{f / f}$ mice since deletion of Bmpr1a with Prrx1-Cre led to embryonic lethality (data not shown). Three doses of TAM were administered i.p. to 2-month-old Prrx1-CreERT; Bmpr1 $a^{f / f}$ and Bmpr1 $a^{f / f}$ mice. The mice were euthanized 2 months later and the lungs were harvested and analyzed (Figure 2A). Quantitative RT-PCR analysis of lungs revealed

A

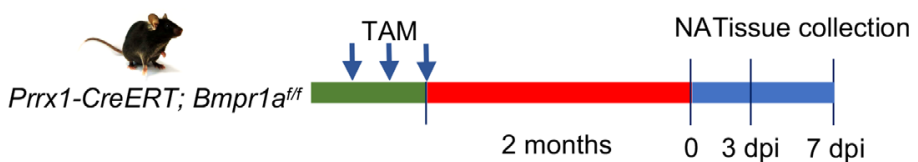

B

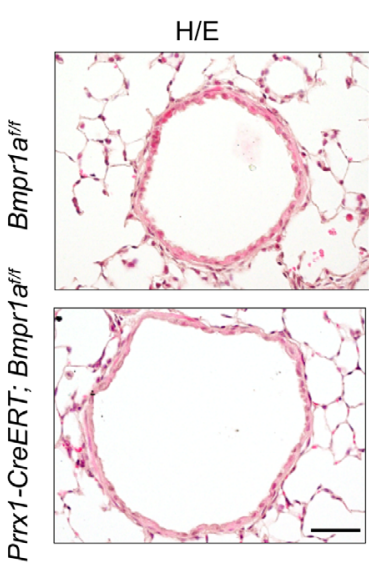

C

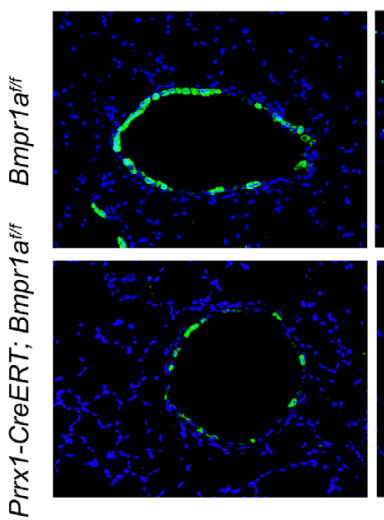

Ki67/ DAPI

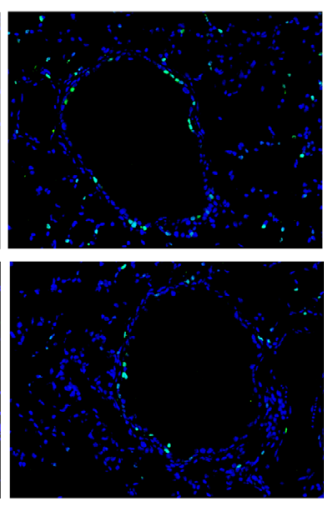

a-Tubulin / DAPI

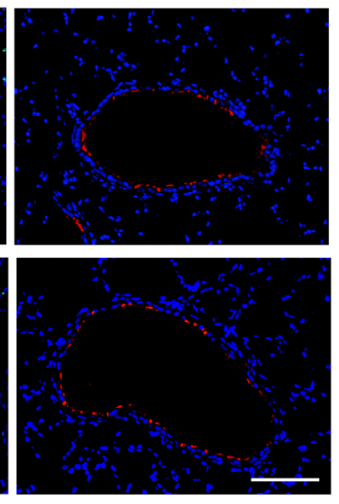

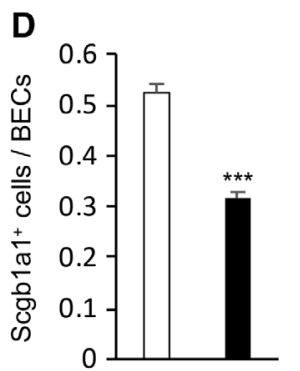
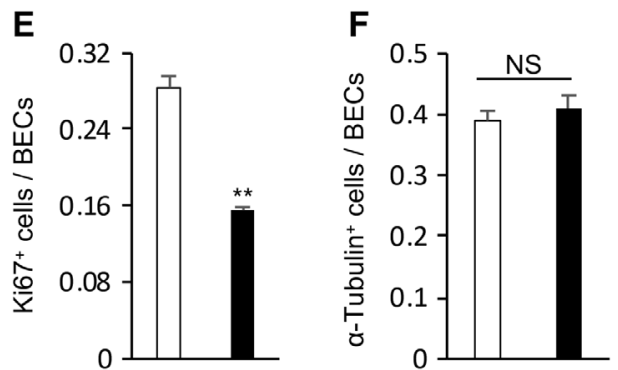

FIGURE 3 BMPR1A was crucial for club cell regeneration. A, Schematic of NA injury and tissue collection following TAM treatment to 2-month-old Prrx1-CreERT; Bmpr1 $a^{f / f}$ and control mice. B, H\&E staining of the lung sections of Prrx1-CreERT; Bmpr1 $a^{f / f}$ and Bmpr1 $a^{f / f}$ mice 7 days after NA injury revealed bronchiolar structure defects in the mutant mice. Scale bar $=50 \mu \mathrm{m}$. C, Immunostaining of Scgb1a1 (left panel), Ki67 (middle panel), and $\alpha$-Tubulin (right panel) 7 days after NA administration. Nuclei were counterstained with DAPI. Scale bar $=50 \mu \mathrm{m}$. D-F, Quantification of Scgb1a1 ${ }^{+}$cells (D), Ki67 ${ }^{+}$cells (E), and $\alpha$-Tubulin ${ }^{+}$cells (F) per total bronchiolar epithelial cells (BECs) 7 days after NA administration. For each lung section, eight different fields were quantified and averaged ( $\mathrm{n}=6$ mice for each genotype). ${ }^{* * *} p<.001 ;{ }^{* *} p<.01$. NS, not significant 
a significant loss of Bmpr1a mRNA in the mutant mice (Figure 2B). Western blot also confirmed a marked decrease in the level of $p$-Smad $1 / 5 / 8$ as well as p-Tak1 and p-p38MAPKs in the lung samples of the mutant mice (Figure $2 \mathrm{C}$ ). These results indicate that activation of Tak1-p38Mapk pathway, which is often affected by multiple signaling pathways, is largely controlled by BMPs in bronchiolar epithelial cells. In addition, it appeared that three doses of TAM successfully induced the deletion of Bmpr1a. In comparison with the control littermates, the Prrx1-CreERT; Bmpr1a $a^{f / f}$ mice displayed a moderate increase in the numbers of Scgb1a1 ${ }^{+}$club cells but no significant change in the number of ciliated cells (Figure 2D-G), suggesting that ablation of Bmpr1a had a modest effect on the homeostasis of adult bronchiolar epithelium. This is in contrast to the critical roles BMPR1A played in lung development since ablating Bmpr1a has been shown to disrupt branching morphogenesis and airway formation in the lung and cause neonatal respiratory distress. ${ }^{4,19,44}$ In addition, Bmpr1a ablation in the epithelium by Sftpc-Cre resulted in defective lung development, accompanied by reduction in epithelium proliferation and increased apoptosis. $^{4}$

A

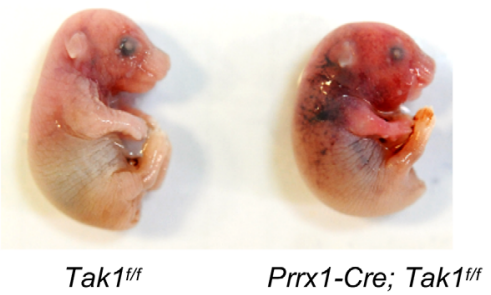

C
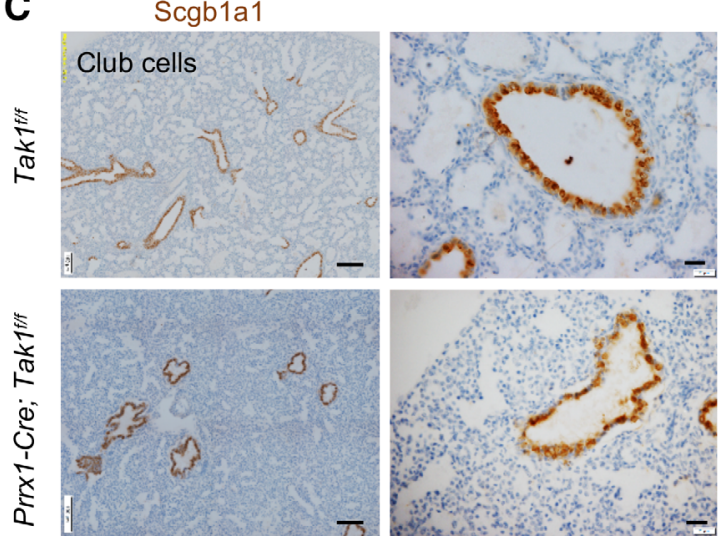

\section{5 | Bmpr1a ablation disrupted club cell regeneration}

We then examined the effect of Bmpr1a deletion on regeneration of bronchiolar epithelium. Adult Prrx1-CreERT; Bmpr1a $a^{f / f}$ and control mice were given a single dose of NA following three doses of TAM administration. The lungs were harvested 3 or 7 days after NA exposure and processed for immunostaining (Figure $3 \mathrm{~A}$ ). We found that deletion of Bmpr1a impaired the repair of bronchiolar epithelium at both time points. Three days after NA exposure, the number of Scgb1a1 $1^{+}$club cells present on the bronchioles was significantly reduced in the knockout mice (Figure S2A-C). This was likely due to the impaired proliferation of the club cells since terminal bronchioles of Bmpr1a ablated mice contained fewer $\mathrm{Ki} 67^{+}$proliferative cells than those of control mice (Figure S2B,D). The defects in bronchiolar epithelium repair became more prominent at day 7 after NA exposure in the mutant mice, as manifested by decreased numbers of Scgb1a1 $1^{+}$club cells and $\mathrm{Ki} 67^{+}$cells (Figure 3B-E). However, we did not detect any notable changes in the number of ciliated cells

B

$H / E$
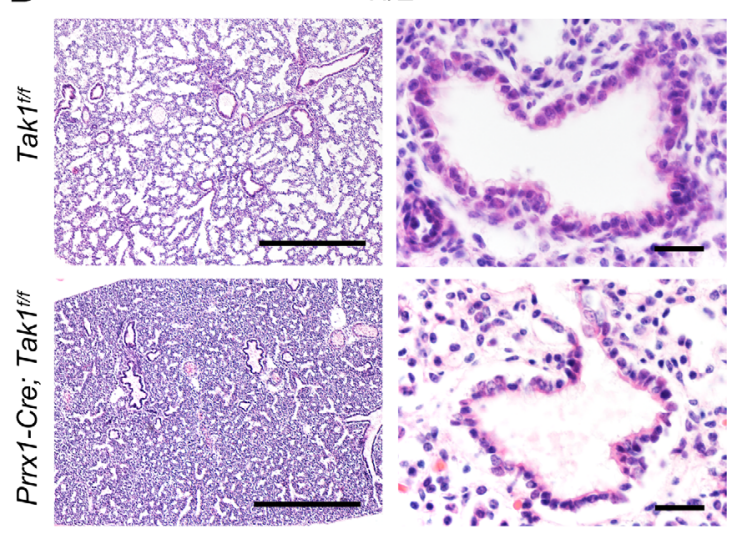

D
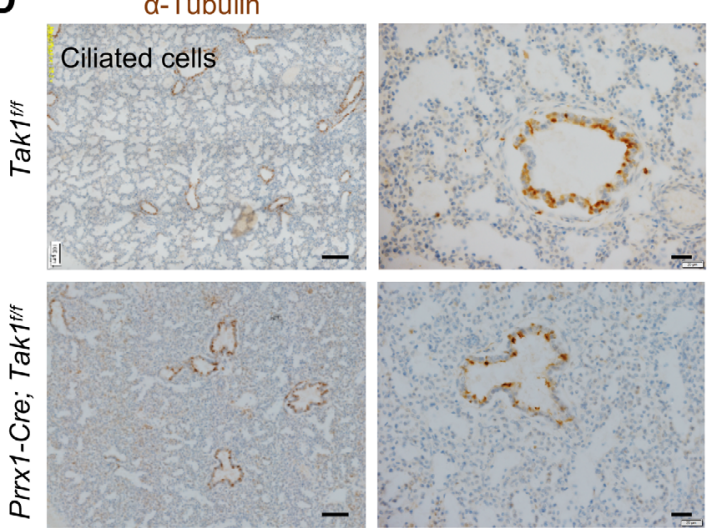

FIGURE 4 Postnatal lethality with delayed bronchiole development resulted from Tak1 ablation in Prrx1 ${ }^{+}$cells. A, Newborn Prrx1-Cre; Tak1 ${ }^{f / f}$ mice died shortly after birth. Newborn pups' macrograph. B, H\&E staining revealed morphological differences in the Tak1 knockout and control mouse lung sections. Scale bars $=500 \mu \mathrm{m}$ (left panel) and $50 \mu \mathrm{m}$ (right panel). C, Immunohistochemistry of Scgb1a1 for club cells. Lower magnification is provided on the left panel and higher magnification is on the right panel. Sections were developed using DAB chromogen and nuclei were counterstained with hematoxylene. Scale bars $=100 \mu \mathrm{m}$ (left panel) and $20 \mu \mathrm{m}$ (right panel). D, Immunohistochemistry of $\alpha$-Tubulin for ciliated cells. Lower magnification is provided on the left panel and higher magnification is on the right panel. Sections were developed using DAB chromogen and nuclei were counterstained with hematoxylene. Scale bars $=100 \mu \mathrm{m}$ (left panel) and $20 \mu \mathrm{m}$ (right panel) 
either 3 days (Figure S2B,E) or 7 days after NA treatment (Figure 3C,F). These results indicate that BMPR1A is crucial for club cell regeneration.

\section{6 | Tak1 deletion in Prrx1 ${ }^{+}$cells led to defective bronchiole and neonatal lethality}

We then tested the physiological functions of Tak1 in lung development using Prrx1-Cre; Tak1 mice since Tak1 was shown to be activated in bronchiolar epithelial cells during homeostasis in a BMPR1A-dependent manner (Figure S1A and Figure 2C). We found that Prrx1-Cre; Tak1 $1^{f / f}$ pups died shortly after birth. Histological analysis revealed some anatomical abnormalities in the lungs of knockout pups (Figure 4A). When H/E stained lung sections were examined, we found that the knockout mouse lung was morphologically different from the control. The bronchiolar and alveolar areas were not well differentiated in the knockout mice compared with those of control mice (Figure 4B, left panel). H\&E staining also showed that the bronchioles in the knockout mice were poorly organized (Figure 4B, right panel). Immunohistochemistry staining confirmed that Scgb1a $1^{+}$club cells and $\alpha$-Tubulin ${ }^{+}$ciliated cells were less differentiated in the mutant mice (Figure 4C,D). To our understanding, the development of the bronchiolar epithelium in Prrx1-Cre; Tak1 ${ }^{f / f}$ newborn pups were delayed compared with the control littermates. These results, for the first time, show that Tak1 is required for the development of bronchiolar epithelium.

A
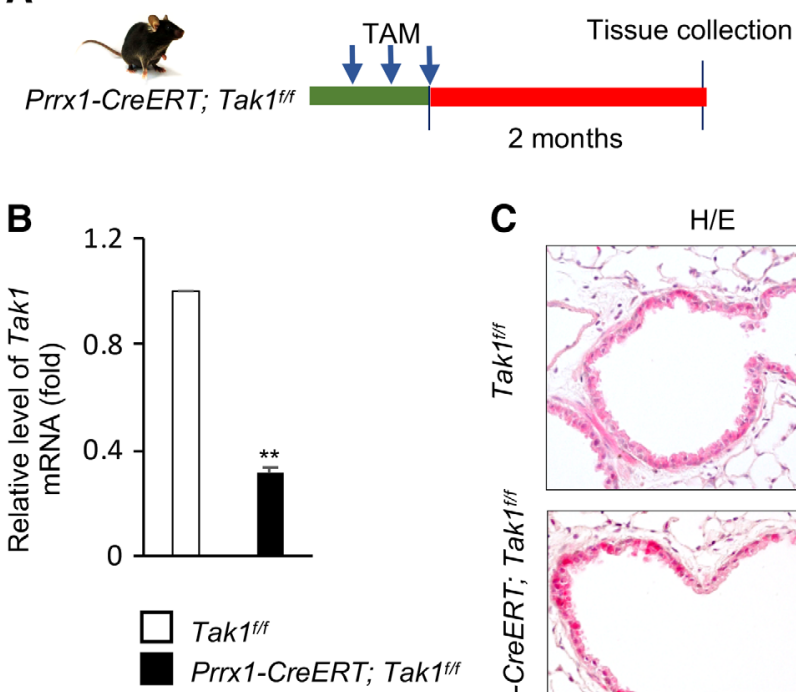

C $\quad \mathrm{H} / \mathrm{E}$

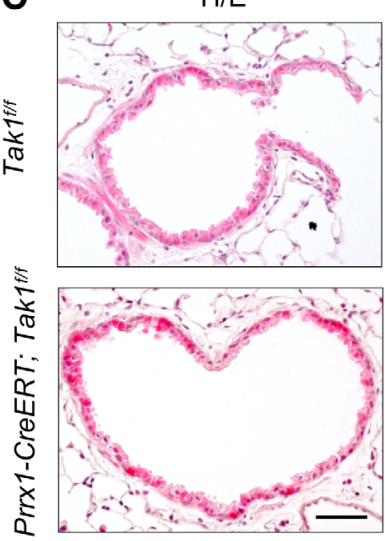

D

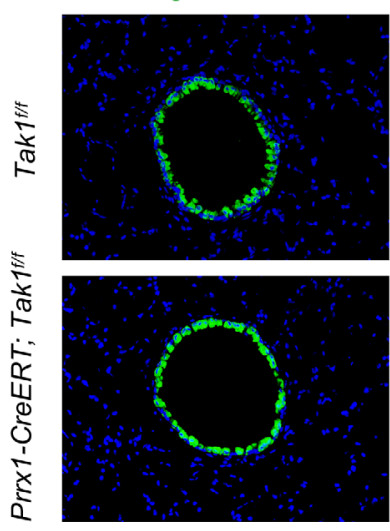

a-Tubulin/ DAP

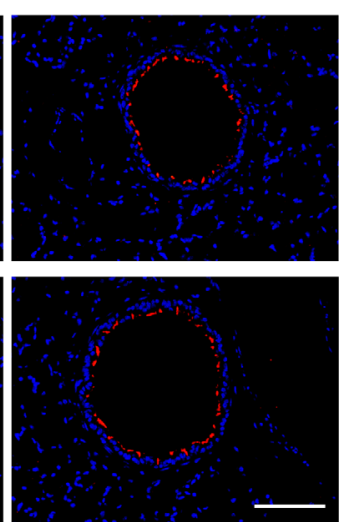

E

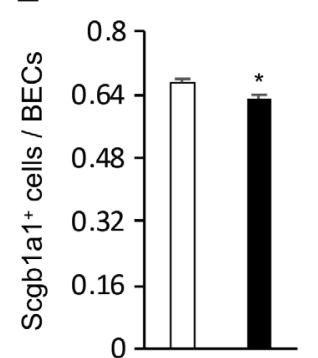

Tak $1^{f / f}$

Prrx1-CreERT; Tak1fff

FIGURE 5 Tak1 ablation in Prrx1 ${ }^{+}$cells resulted in a minor defect in club cell homeostasis. A, Schematic of TAM treatment of 2-month-old Prrx1-CreERT; Tak1 ${ }^{f / f}$ and Tak1 ${ }^{f / f}$ (control) mice. B, qPCR analysis illustrates the successful deletion of Tak1 gene after TAM administration $(\mathrm{n}=6$ mice for each genotype). ${ }^{* *} p<.01$. C, H\&E staining of Tak1 knockout and control lung sections after TAM administration. Scale bar $=50 \mu \mathrm{m}$. D, Immunostaining of Scgb1a1 (left panel) and $\alpha$-Tubulin (right panel) of Tak1 ablated and control lung sections after three doses of TAM. Nuclei were counterstained with DAPI. Scale bar $=50 \mu \mathrm{m}$. E,F, Quantification of Scgb1a1 ${ }^{+}$cells (E) and $\alpha$-Tubulin ${ }^{+}$cells (F) per total bronchiolar epithelial cells. For each lung section, eight different fields were quantified and averaged ( $n=6$ mice for each genotype). ${ }^{*} p<.05$ 


\section{7 | Ablation of Tak1 disrupted club cell regeneration}

To determine the functions of Tak1 in Prrx $1^{+}$cells in adult mice, we generated Prrx1-CreERT; Tak1 ${ }^{f / f}$ mice. Three doses of TAM were administered to 2-month-old Prrx1-CreERT; Tak1 ${ }^{f / f}$ and Tak1 ${ }^{f / f}$ mice. Two months later, the mice were euthanized and the lungs were harvested for analyses (Figure 5A). Quantitative RT-PCR revealed a significant loss of Tak1 mRNA in the mutant mice compared with control littermates (Figure 5B). Depletion of Tak1 led to a modest decrease in number of Scgb1a1 $1^{+}$club cells and a modest increase in the number $\alpha$-Tubulin ${ }^{+}$ciliated cells (Figure 5C-F).

Next, following TAM administration, the Prrx1-CreERT; Tak1 ${ }^{f / f}$ and control mice were exposed to NA and the lungs were harvested 3 and
FIGURE 6 Ablation of Tak1 in Prrx $1^{+}$cells resulted in severe defect in club cell regeneration. A, Schematic of NA administration and tissue collection following TAM treatment to 2-monthold Prrx1-CreERT; Tak1 ${ }^{f / f}$ and control mice. $B, H \& E$ staining of the lung sections of Prrx1-CreERT; Tak1 ${ }^{f / f}$ and Tak ${ }^{f / f}$ mice 7 days after NA administration revealed bronchiolar structure defects in the mutant mice. Scale bar $=50 \mu \mathrm{m}$. C, Immunostaining of Scgb1a1 (left panel), Ki67 (middle panel), and $\alpha$-Tubulin (right panel) on lung sections of Tak1 knockout and control mice 7 days after NA injury. Nuclei were counterstained with DAPI. Scale bar $=50 \mu \mathrm{m}$. D-F, Quantification of Scgb1a1 $1^{+}$cells (D), Ki67 ${ }^{+}$cells (E), and $\alpha$-Tubulin ${ }^{+}$cells (F) per total bronchiolar epithelial cells 7 days following NA exposure. For each lung section, eight different fields were quantified and averaged ( $\mathrm{n}=6$ mice for each genotype). ${ }^{* *} p<.01$;

${ }^{* * *} p<.001$. G, Immunohistochemistry of $p$-p38MAPKs on lung sections of Prrx1-CreERT; Tak1 ${ }^{f / f}$ and control mice 3 days (left panel) and 7 days (right panel) after NA injury. Sections were developed using DAB chromogen and nuclei were counterstained with hematoxylene. Scale bar $=50 \mu \mathrm{m} . \mathrm{H}, \mathrm{I}$, Western blot analysis detected significant decreases in the levels of p-p38 in the Tak1 deficient mouse lung samples compared to the control 3 days $(\mathrm{H})$ or 7 days (I) after NA administration. Left panel: representative western blot result; right panel: quantification of relative protein expressions plotted in the graph as fold change in protein expression ( $\mathrm{n}=3$ mice for each genotype). ${ }^{*} p<.05$. dpi, day post injury; NS, not significant
A

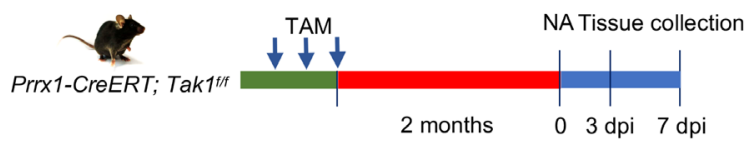

B

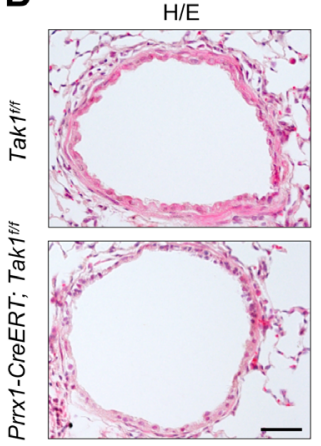

C

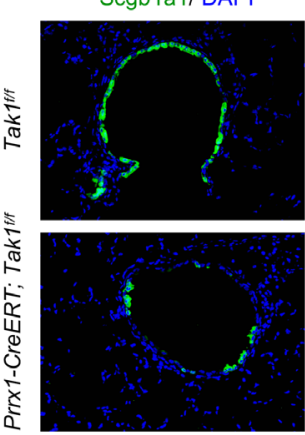

Ki67/ DAPI
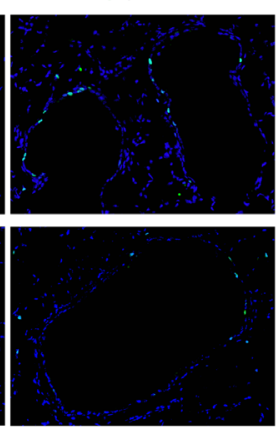

a-Tubulin / DAPI

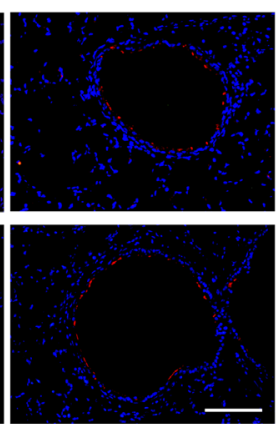

D

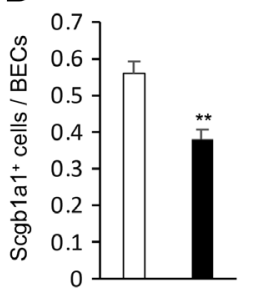

G
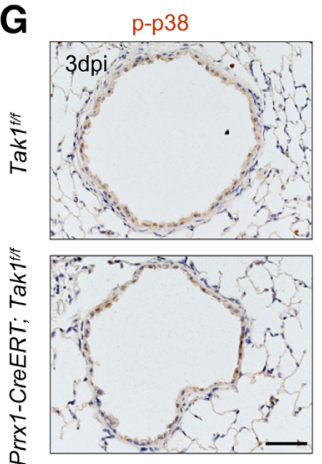

E

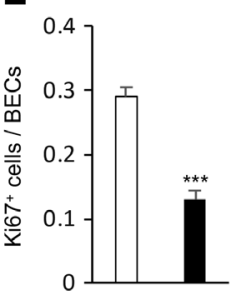

$\mathbf{F}$

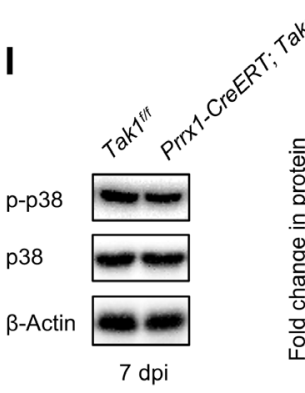

H

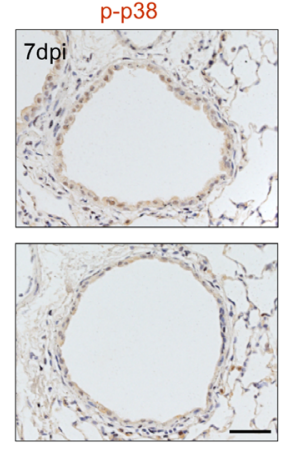

p-p3

p38

$\beta$-Actin

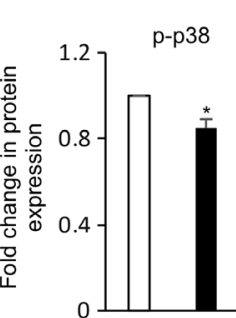

Tak1tir

Prrx1-CreERT; Tak1f/f 
A

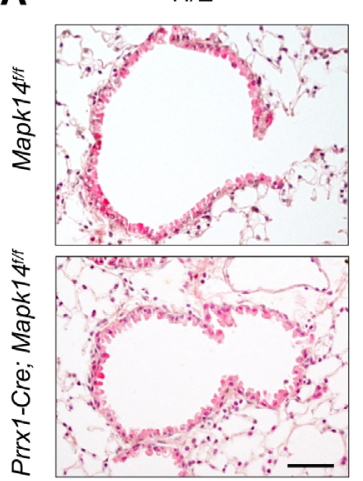

D

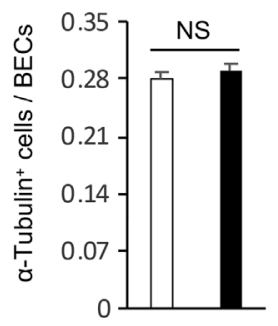

F
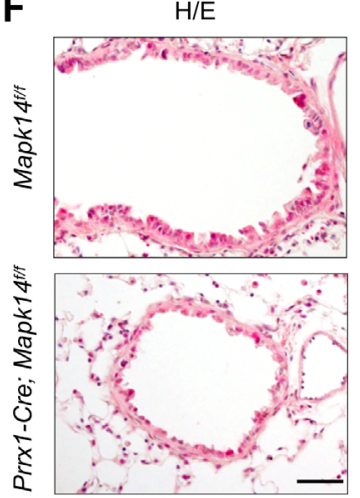

B

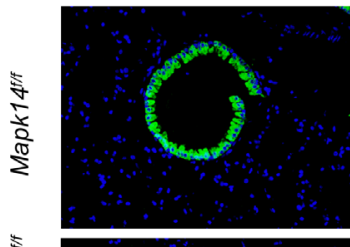

$\frac{0}{2}$

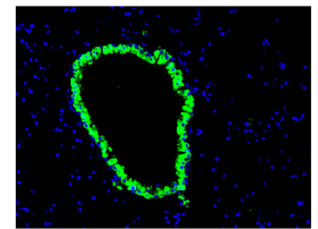

E

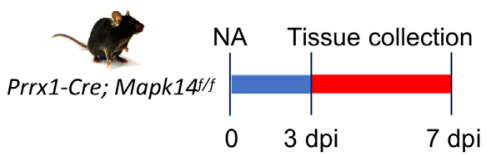

a-Tubulin/ DAPI
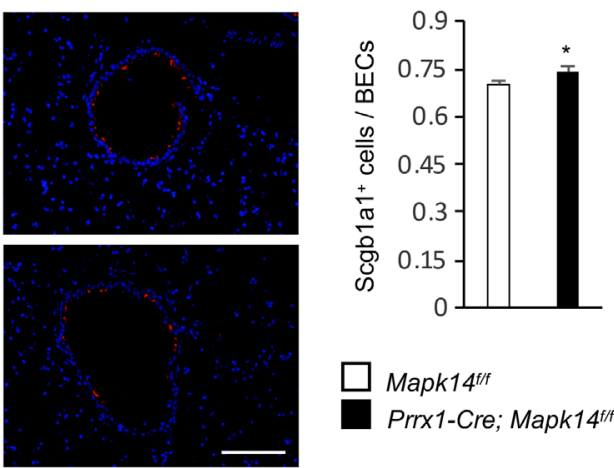

Mapk14t

Prrx1-Cre; Mapk14t/
G
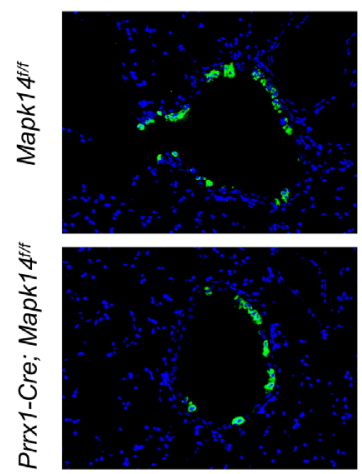

a-Tubulin / DAPI
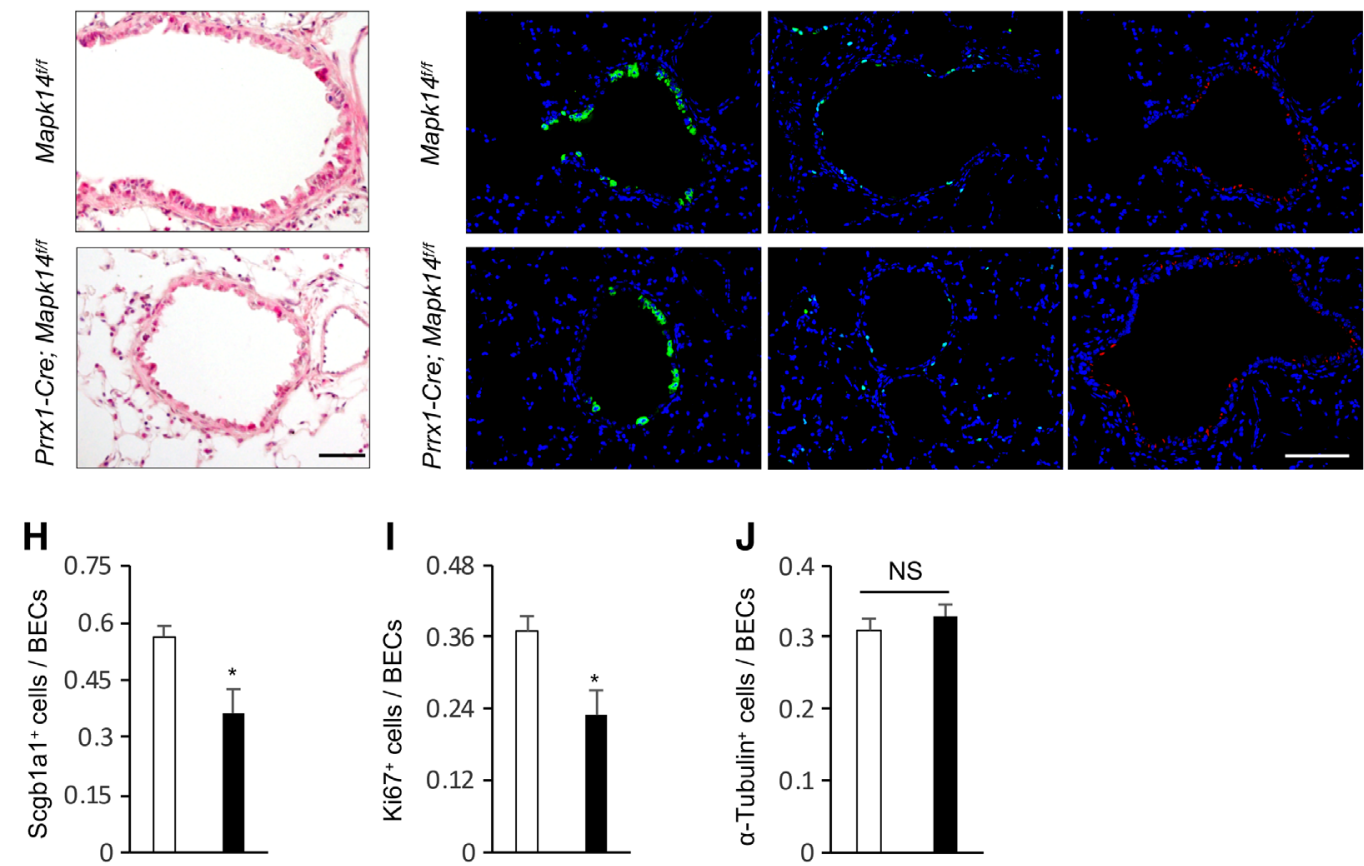

\section{I}
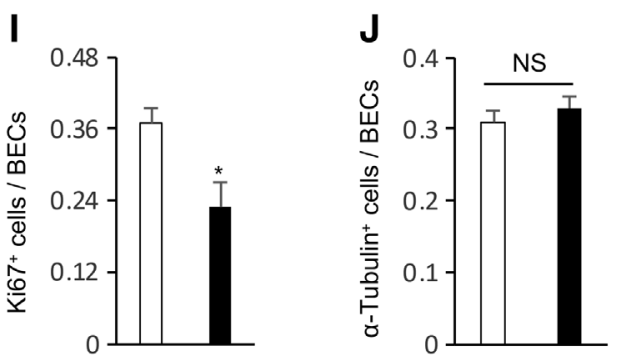

FIGURE 7 Deletion of Mapk14 in Prrx1+ cells impaired regeneration of bronchiolar epithelium. A, H\&E staining of the Prrx1-Cre; Mapk14/f and Mapk14 ${ }^{f / f}$ lung sections revealed little difference. Scale bar $=50 \mu \mathrm{m} . \mathrm{B}$, Immunostaining of Scgb1a1 (left panel) and $\alpha$-Tubulin (right panel). Nuclei were counterstained with DAPI. Scale bar $=50 \mu \mathrm{m}$. C,D, Quantification of Scgb1a1 ${ }^{+}$cells (C) and $\alpha-$ Tubulin ${ }^{+}$cells (D) per total bronchiolar epithelial cells (BECs). For each lung section, eight different fields were quantified and averaged ( $\mathrm{n}=6$ mice for each genotype). ${ }^{*} p<.05$.

E, Schematic of NA administration and tissue collection to Prrx1-Cre; Mapk14 ${ }^{f / f}$ and control mice. F, Histological examination of Mapk14 knockout and control lung sections 7 days after NA exposure revealed bronchiolar structure defects in the mutant mice. Scale bar $=50 \mu \mathrm{m}$. G, Immunostaining of Scgb1a1 (left panel), Ki67 (middle panel), and $\alpha$-Tubulin (right panel) 7 days after NA injury. Nuclei were counterstained with DAPI. Scale bar $=50 \mu \mathrm{m} . \mathrm{H}-\mathrm{J}$, Quantification of Scgb1a1 ${ }^{+}$cells $(\mathrm{H}), \mathrm{Ki} 67^{+}$cells (I), and $\alpha$-Tubulin ${ }^{+}$cells $(\mathrm{J})$ per total bronchiolar epithelial cells 7 days after NA exposure. For each lung section, eight different fields were quantified and averaged ( $\mathrm{n}=6$ mice for each genotype). ${ }^{*} p<.05$. NS, not significant 
7 days later (Figure 6A). Three days after NA-induced injury, we observed a significant delay in regeneration of the bronchiolar epithelium as the numbers of Scgb1a1 $1^{+}$club cells and Ki $67^{+}$proliferating cells were significantly decreased in the knockout mice (Figure S3A$D$ ), whereas the number of ciliated cells was barely changed (Figure S3B,E). Seven days after NA injury, club cell regeneration and proliferation were more severely affected in Tak1 deficient mice (Figure 6B-E). However, no detectable effect on the number of ciliated cells was observed (Figure 6C,F). These findings indicate that Tak1 plays an important role in the Prrx $1^{+}$cells-mediated regeneration and repair of bronchiolar epithelium, similar to BMPR1A.

\section{8 | Mapk14 deletion in Prrx $1^{+}$cells resulted in defects in regeneration}

p38MAPKs are important downstream kinases of Tak1 and are involved in lung epithelial branching morphogenesis. ${ }^{26,45}$ Previous studies have shown that Mapk14, the dominant isoform of p38MAPKs in the lung, plays critical roles in lung stem cells and lung development. ${ }^{28}$ Indeed, immunohistochemistry revealed that the levels of activated p38 were reduced in Tak1 knockout mice after NA exposure (Figure 6G). Western blot analysis confirmed decreased levels of p-p38 in the lung tissues of Tak1 knockout mice 3 and 7 days after NA administration (Figure 6H,I). The modest decease in p-p38 is likely caused by presence of cells not labeled by Prrx1 in the lung. We then generated Prrx1-Cre; Mapk14 ${ }^{f / f}$ mice to determine the functions of Mapk14 in club cells in adult mice. Adult Prrx1-Cre; Mapk14 $4^{f / f}$ mice have normal appearance. The lungs were harvested from the 2.5-month-old mice and analyzed. We found that ablation of Mapk14 in Prrx $1^{+}$cells resulted in a modest increase in the number of Scgb1a1 $1^{+}$club cells, whereas the number of ciliated cells was not affected (Figure 7A-D). These results indicate that Mapk14 plays minimal roles in the development and homeostasis of bronchiolar epithelia.

To determine the functions of Mapk14 in regeneration of bronchiolar epithelial cells, we exposed Prrx1-Cre; Mapk14 f/f mice to NA and the lungs were harvested 3 and 7 days later (Figure 7E). We observed a significant impairment in regeneration/repair of bronchiolar epithelium at day 3 or 7 after NA exposure in the Mapk14 ablated mice (Figure S4A and Figure 7F). At both time points, the numbers of $\mathrm{Scgb} 1 \mathrm{a} 1^{+}$club cells and $\mathrm{Ki} 67^{+}$proliferating cells were decreased in the knockout mice (Figure 7G-I and Figure S4B-D), whereas no significant change in the number of ciliated cells was observed either 3 days (Figure S4B,E) or 7 days (Figure 7G,J) after NA injury. These findings suggest that the Mapk14 plays important roles in Prrx1 lineage cellmediated bronchiole regeneration/repair.

\section{4 | DISCUSSION}

Our lineage tracing experiments based on transgenic Prrx1-Cre and Prrx1-CreERT mice lines show that Prrx1 marks club cells and moreover, these club cells can replenish the bronchiolar epithelium during repair, indicating that Prrx $1^{+}$club cells act as transiently amplifying progenitor cells. This is a surprising finding as Prrx1 is generally believed to be a marker for mesenchymal stem cells, which form bone and cartilage in vivo and can differentiate into osteoblasts, chondrocytes, and adipocytes but not epithelial cells in vitro. ${ }^{46,47}$ Yet, the club cells are epithelial cells in nature and are supposed to be derived from the endoderm. During embryonic development, the primary lung bud is surrounded by ventral mesenchyme, which secretes multiple growth regulators such as Wnt and BMP molecules to direct endodermal cell proliferation and differentiation. ${ }^{16,48}$ One possible explanation is that progenitors of club cells are derived from mesenchymal cells via a process termed mesenchymal-epithelial transition. Another possibility is that Prrx1, the homeobox transcription factor, may play an important role in maintaining the stemness of multiple cell lineages including mesenchymal cells and club cells. A similar example is Lgr5, which is established as a stem cell marker for gastrointestinal epithelial cells but has recently been reported to mark lung fibroblasts. ${ }^{49}$ Similarly, club cells are a population of progenitor cells comprising of highly heterogenous subsets. Besides Scgb1a1, club cells marked by Upk3a or Id2 have been reported. ${ }^{8,50}$ Although Prrx1 lineage cells do express Scgb1a1 during homeostasis and regeneration, further investigation is needed to examine the relationships among Prrx1 lineage cells and Upk3a-expressing club cells and Id2-marked multipotent club cell progenitors.

BMPs play vital roles in lung development and homeostasis. These factors are supplied by mesenchymal cells and they regulate both cell proliferation and differentiation of lung epithelial cells. As such, disruption of BMP-Smad1 signaling impairs lung development. ${ }^{19,21,22}$ Moreover, BMPs have been shown to play important roles in lung repair mediated by basal cells and AEC2. The major function of BMPs appears to inhibit cell proliferation in steady-state condition. Upregulation of BMP inhibitors during injury may promote proliferation of basal cells, AEC2 cells and thus, facilitates the repair process. $^{31,32}$ However, here, we show that the ablation of Bmpr1a resulted in defective regeneration of bronchiolar epithelium. In particular, club cell proliferation and expansion are suppressed in the absence of Bmpr1a. Thus, BMPR1A appears to play opposing roles in regeneration of club cells and basal cells and AEC2. ${ }^{32}$ On the other hand, repair of adult lung epithelia is reminiscent of the lung developmental process, and from our results it is evident that ablation of Bmpr1a affects these two processes in a similar fashion. Loss of BMP signaling causes developmental defects associated with impaired epithelial proliferation. For example, inactivation of Bmpr1a and Bmpr1b results in tracheal agenesis ${ }^{16}$ and Bmpr1a ablation in lung epithelial cells resulted in defective lung development, accompanied by reduction in epithelial proliferation and extensive apoptosis. ${ }^{4}$

Moreover, the effects of Bmpr1a ablation on bronchiolar epithelium regeneration are reproduced by ablation of Tak1 or Mapk14, which are activated in club cells in a BMPR1A-dependent manner. Although Tak1 is known to promote cell proliferation and survival, ${ }^{51,52}$ activation of p38MAPKs often inhibits cell proliferation, in particular after activation by stress and cytokines. ${ }^{53}$ Indeed, it has been reported that p38MAPKs promote the differentiation but inhibits the proliferation of lung stem cells in a mouse model in which Mapk14 was deleted using a CreERT driven by RERTn, which encodes the large subunit of RNA polymerase II. ${ }^{28}$ On the other hand, there are examples where p38MPAKs have 
been shown to promote cell proliferation. ${ }^{54,55} \mathrm{~A}$ recent study also reported that Notch1 is required for club cell regeneration. ${ }^{56}$ Since Notch1 signaling crosstalks with p38MAPKs, ${ }^{57,58}$ future studies will be needed to test their functional link in club cell regeneration. Nevertheless, our genetic evidence indicates that both Tak1 and p38MAPKs play positive roles in club cell proliferation and expansion during regeneration.

The present study also sheds light on the function of the Tak1p38MAPK pathway in lung development. We found that Tak1 deletion in Prrx1-expressing cells led to delayed development of bronchiolar epithelium with the mice dying right after birth. However, we found that the ablation of Mapk14 from Prrx $1^{+}$lineage cells did not cause obvious phenotypes in the bronchiolar epithelium. This is in contrast with previous studies showing that Mapk14 is essential for lung development, especially in lung stem cells. ${ }^{28}$ One possible explanation is that the same signal may have different effects on stem/progenitor cells located at different sites of the airways, which certainly warrants further investigation.

\section{5 | CONCLUSION}

Our genetic lineage tracing and gene knockout studies indicate that club cells in bronchiole can be marked by homeobox transcription factor Prrx1. Moreover, the BMPR1A-Tak1-p38MAPK pathway plays critical roles in club cell regeneration. Since club cells constitute about $70 \%$ of cells in the terminal bronchiolar epithelium, which is also a major site for the development of various lung diseases, our study identifies BMPR1A-Tak1-p38MAPK pathway as a potential clinical target to treat bronchiole-related disorders.

\section{ACKNOWLEDGMENTS}

The work was supported by the National Key Scientific Program (2018YFA0800803 and 2017YFA0103602), National Natural Science Foundation of China (81130039 and 81421061), and also by the Grants from the State Key Laboratory of Oncogenes and Related Genes No. 90-17-03.

\section{CONFLICT OF INTEREST}

The authors indicated no potential conflicts of interest.

\section{AUTHOR CONTRIBUTIONS}

M.S.: conception and design, conducting experiments, collection and/ or assembly of data, data analysis and interpretation, and manuscript writing; B.L.: conception and design, data analysis and interpretation, manuscript writing, and manuscript revision; H.L.: conception and design, manuscript revision, and final approval of manuscript; S.B.: conducting experiments; collection and/or assembly of data, and manuscript revision; P.L.: conducting experiments; Y.M.: provision of study material.

\section{DATA AVAILABILITY STATEMENT}

Data supporting the findings of this study are included within the article and available upon request to the corresponding author (liuhj@sjtu.edu.cn).

\section{ORCID}

Md Shafiquzzaman (D) https://orcid.org/0000-0002-4668-0983

Soma Biswas (D) https://orcid.org/0000-0002-1427-2678

Huijuan Liu (D) https://orcid.org/0000-0002-8801-4815

\section{REFERENCES}

1. Herriges M, Morrisey EE. Lung development: orchestrating the generation and regeneration of a complex organ. Development. 2014;141 (3):502-513.

2. Hogan BL, Barkauskas CE, Chapman HA, et al. Repair and regeneration of the respiratory system: complexity, plasticity, and mechanisms of lung stem cell function. Cell Stem Cell. 2014;15(2):123-138.

3. Metzger RJ, Klein OD, Martin GR, Krasnow MA. The branching programme of mouse lung development. Nature. 2008;453(7196): 745-750.

4. Eblaghie MC, Reedy M, Oliver T, Mishina Y, Hogan BLM. Evidence that autocrine signaling through Bmpr1a regulates the proliferation, survival and morphogenetic behavior of distal lung epithelial cells. Dev Biol. 2006;291(1):67-82.

5. Rock JR, Gao X, Xue Y, Randell SH, Kong YY, Hogan BLM. Notchdependent differentiation of adult airway basal stem cells. Cell Stem Cell. 2011;8(6):639-648.

6. Guha A, Vasconcelos M, Cai Y, et al. Neuroepithelial body microenvironment is a niche for a distinct subset of Clara-like precursors in the developing airways. Proc Natl Acad Sci USA. 2012;109(31):1259212597.

7. Kumar PA, Hu Y, Yamamoto $\mathrm{Y}$, et al. Distal airway stem cells yield alveoli in vitro and during lung regeneration following H1N1 influenza infection. Cell. 2011;147(3):525-538.

8. Rawlins EL, Okubo T, Xue Y, et al. The role of Scgb1a1+ Clara cells in the long-term maintenance and repair of lung airway, but not alveolar, epithelium. Cell Stem Cell. 2009;4(6):525-534.

9. Reynolds SD, Hong KU, Giangreco A, et al. Conditional Clara cell ablation reveals a self-renewing progenitor function of pulmonary neuroendocrine cells. Am J Physiol Lung Cell Mol Physiol. 2000;278(6): L1256-L1263.

10. Tata PR, Mou H, Pardo-Saganta A, et al. Dedifferentiation of committed epithelial cells into stem cells in vivo. Nature. 2013;503(7475): 218-223.

11. Watson JK, Rulands S, Wilkinson AC, et al. Clonal dynamics reveal two distinct populations of basal cells in slow-turnover airway epithelium. Cell Rep. 2015;12(1):90-101.

12. Guha A, Deshpande A, Jain A, Sebastiani P, Cardoso WV. Uroplakin $3 a(+)$ cells are a distinctive population of epithelial progenitors that contribute to airway maintenance and post-injury repair. Cell Rep. 2017;19(2):246-254.

13. Green MD, Huang SXL, Snoeck HW. Stem cells of the respiratory system: from identification to differentiation into functional epithelium. Bioessays. 2013;35(3):261-270.

14. Kajstura J, Rota M, Hall SR, et al. Evidence for human lung stem cells. N Engl J Med. 2011;364(19):1795-1806.

15. Bellusci S, Henderson R, Winnier G, Oikawa T, Hogan BL. Evidence from normal expression and targeted misexpression that bone 
morphogenetic protein (BMP-4) plays a role in mouse embryonic lung morphogenesis. Development. 1996;122(6):1693-1702.

16. Domyan ET, Ferretti E, Throckmorton K, Mishina Y, Nicolis SK, Sun $X$. Signaling through BMP receptors promotes respiratory identity in the foregut via repression of Sox2. Development. 2011; 138(5):971-981.

17. Geng $\mathrm{Y}$, Dong $\mathrm{Y}, \mathrm{Yu} \mathrm{M}$, et al. Follistatin-like 1 (Fstl1) is a bone morphogenetic protein (BMP) 4 signaling antagonist in controlling mouse lung development. Proc Natl Acad Sci USA. 2011;108(17): 7058-7063.

18. Li Q, Jiao J, Li H, et al. Histone arginine methylation by Prmt5 is required for lung branching morphogenesis through repression of BMP signaling. J Cell Sci. 2018;131(14):jcs217406.

19. Weaver M, Yingling JM, Dunn NR, Bellusci S, Hogan BL. Bmp signaling regulates proximal-distal differentiation of endoderm in mouse lung development. Development. 1999;126(18):4005-4015.

20. Heldin $\mathrm{CH}$, Miyazono $\mathrm{K}$, ten Dijke P. TGF-beta signalling from cell membrane to nucleus through SMAD proteins. Nature. 1997; 390(6659):465-471.

21. Chen $\mathrm{C}$, Chen $\mathrm{H}$, Sun J, et al. Smad1 expression and function during mouse embryonic lung branching morphogenesis. Am J Physiol Lung Cell Mol Physiol. 2005;288(6):L1033-L1039.

22. $\mathrm{Xu} \mathrm{B}$, Chen $\mathrm{C}$, Chen $\mathrm{H}$, et al. Smad1 and its target gene Wif1 coordinate BMP and Wnt signaling activities to regulate fetal lung development. Development. 2011;138(5):925-935.

23. Kua HY, Liu H, Leong WF, et al. c-Abl promotes osteoblast expansion by differentially regulating canonical and non-canonical BMP pathways and p16INK4a expression. Nat Cell Biol. 2012;14(7):727-737.

24. Shibuya $H$, Iwata $H$, Masuyama $N$, et al. Role of TAK1 and TAB1 in BMP signaling in early Xenopus development. EMBO J. 1998;17(4): 1019-1028.

25. Shim JH, Greenblatt MB, Xie M, et al. TAK1 is an essential regulator of BMP signalling in cartilage. EMBO J. 2009;28(14):2028-2041.

26. Liu Y, Martinez L, Ebine K, Abe MK. Role for mitogen-activated protein kinase p38 alpha in lung epithelial branching morphogenesis. Dev Biol. 2008;314(1):224-235.

27. Hui L, Bakiri L, Mairhorfer A, et al. p38alpha suppresses normal and cancer cell proliferation by antagonizing the JNK-c-Jun pathway. Nat Genet. 2007;39(6):741-749.

28. Ventura JJ, Tenbaum S, Perdiguero E, et al. p38alpha MAP kinase is essential in lung stem and progenitor cell proliferation and differentiation. Nat Genet. 2007;39(6):750-758.

29. Myllarniemi M, Lindholm P, Ryynänen $M J$, et al. Gremlin-mediated decrease in bone morphogenetic protein signaling promotes pulmonary fibrosis. Am J Respir Crit Care Med. 2008;177(3):321-329.

30. Rosendahl A, Pardali E, Speletas M, ten Dijke P, Heldin CH, Sideras P. Activation of bone morphogenetic protein/Smad signaling in bronchial epithelial cells during airway inflammation. Am J Respir Cell Mol Biol. 2002;27(2):160-169.

31. Tadokoro T, Gao X, Hong CC, Hotten D, Hogan BLM. BMP signaling and cellular dynamics during regeneration of airway epithelium from basal progenitors. Development. 2016;143(5):764-773.

32. Chung MI, Bujnis M, Barkauskas CE, Kobayashi Y, Hogan BLM. Niche-mediated BMP/SMAD signaling regulates lung alveolar stem cell proliferation and differentiation. Development. 2018;145(9): $\operatorname{dev} 163014$

33. Barnes PJ. Senescence in COPD and its comorbidities. Annu Rev Physiol. 2017;79:517-539.

34. Sutherland KD, Berns A. Cell of origin of lung cancer. Mol Oncol. 2010;4(5):397-403.

35. Kawanami A, Matsushita T, Chan YY, Murakami S. Mice expressing GFP and CreER in osteochondro progenitor cells in the periosteum. Biochem Biophys Res Commun. 2009;386(3):477-482.
36. Mishina Y, Hanks MC, Miura S, Tallquist MD, Behringer RR. Generation of Bmpr/Alk3 conditional knockout mice. Genesis. 2002;32(2): 69-72.

37. Li P, Deng Q, Liu J, et al. Roles for HB-EGF in mesenchymal stromal cell proliferation and differentiation during skeletal growth. J Bone Miner Res. 2019;34(2):295-309.

38. Logan M, Martin JF, Nagy A, Lobe C, Olson EN, Tabin CJ. Expression of Cre Recombinase in the developing mouse limb bud driven by a Prxl enhancer. Genesis. 2002;33(2):77-80.

39. $\mathrm{Wu} \mathrm{H}, \mathrm{Wu} \mathrm{Z}$, Li P, et al. Bone size and quality regulation: concerted actions of mTOR in mesenchymal stromal cells and osteoclasts. Stem Cell Rep. 2017;8(6):1600-1616.

40. Ihida-Stansbury K, McKean DM, Gebb SA, et al. Paired-related homeobox gene Prx1 is required for pulmonary vascular development. Circ Res. 2004;94(11):1507-1514.

41. Martin JF, Bradley A, Olson EN. The paired-like homeo box gene MHox is required for early events of skeletogenesis in multiple lineages. Genes Dev. 1995;9(10):1237-1249.

42. Hong KU, Reynolds SD, Giangreco A, Hurley CM, Stripp BR. Clara cell secretory protein-expressing cells of the airway neuroepithelial body microenvironment include a label-retaining subset and are critical for epithelial renewal after progenitor cell depletion. Am J Respir Cell Mol Biol. 2001;24(6):671-681.

43. Sountoulidis A, Stavropoulos A, Giaglis $S$, et al. Activation of the canonical bone morphogenetic protein (BMP) pathway during lung morphogenesis and adult lung tissue repair. PLoS One. 2012;7(8): e41460.

44. Sun J, Chen H, Chen $\mathrm{C}$, et al. Prenatal lung epithelial cell-specific abrogation of Alk3-bone morphogenetic protein signaling causes neonatal respiratory distress by disrupting distal airway formation. Am J Pathol. 2008;172(3):571-582.

45. Wu X, Zhang W, Font-Burgada J, et al. Ubiquitin-conjugating enzyme Ubc13 controls breast cancer metastasis through a TAK1-p38 MAP kinase cascade. Proc Natl Acad Sci USA. 2014;111 (38):13870-13875.

46. Biswas $\mathrm{S}, \mathrm{Li} \mathrm{P}, \mathrm{Wu} \mathrm{H}$, et al. BMPRIA is required for osteogenic differentiation and RANKL expression in adult bone marrow mesenchymal stromal cells. Sci Rep. 2018;8(1):8475.

47. Cong Q, Jia H, Biswas $\mathrm{S}$, et al. p38alpha MAPK regulates lineage commitment and OPG synthesis of bone marrow stromal cells to prevent bone loss under physiological and pathological conditions. Stem Cell Rep. 2016;6(4):566-578.

48. Que J, Okubo T, Goldenring JR, et al. Multiple dose-dependent roles for Sox2 in the patterning and differentiation of anterior foregut endoderm. Development. 2007;134(13):2521-2531.

49. Lee JH, Tammela T, Hofree M, et al. Anatomically and functionally distinct lung mesenchymal populations marked by Lgr5 and Lgr6. Cell. 2017;170(6):1149-1163.e1112.

50. Rawlins EL, Clark CP, Xue Y, Hogan BLM. The Id2+ distal tip lung epithelium contains individual multipotent embryonic progenitor cells. Development. 2009;136(22):3741-3745.

51. Mihaly SR, Ninomiya-Tsuji J, Morioka S. TAK1 control of cell death. Cell Death Differ. 2014;21(11):1667-1676.

52. Ogura Y, Hindi SM, Sato S, Xiong G, Akira S, Kumar A. TAK1 modulates satellite stem cell homeostasis and skeletal muscle repair. Nat Commun. 2015;6:10123.

53. Oeztuerk-Winder F, Ventura JJ. The many faces of p38 mitogenactivated protein kinase in progenitor/stem cell differentiation. Biochem J. 2012;445(1):1-10.

54. Matsumoto T, Turesson I, Book M, Gerwins P, Claesson-Welsh L. p38 MAP kinase negatively regulates endothelial cell survival, proliferation, and differentiation in FGF-2-stimulated angiogenesis. J Cell Biol. 2002;156(1):149-160. 
55. Wada M, Canals D, Adada M, et al. P38 delta MAPK promotes breast cancer progression and lung metastasis by enhancing cell proliferation and cell detachment. Oncogene. 2017;36(47):6649-6657.

56. Xing Y, Li A, Borok Z, Li C, Minoo P. NOTCH1 is required for regeneration of Clara cells during repair of airway injury. STEM CELLS. 2012; 30(5):946-955.

57. Androutsellis-Theotokis A, Leker RR, Soldner F, et al. Notch signalling regulates stem cell numbers in vitro and in vivo. Nature. 2006; 442(7104):823-826.

58. Cook BD, Evans T. BMP signaling balances murine myeloid potential through SMAD-independent p38MAPK and $\mathrm{NOTCH}$ pathways. Blood. 2014;124(3):393-402.

\section{SUPPORTING INFORMATION}

Additional supporting information may be found online in the Supporting Information section at the end of this article.

How to cite this article: Shafiquzzaman M, Biswas S, Li P, Mishina Y, Li B, Liu H. The noncanonical BMP signaling pathway plays an important role in club cell regeneration. Stem Cells. 2020;38:437-450. https://doi.org/10.1002/stem.3125 